\title{
Stress Biomarkers: Associated with Cognitive Decline and Administered Anesthesia Type in Total Knee Arthroplasties?
}

\section{TO THE EdiTOR:}

With great interest we have read the manuscript written by Dr. Edipoglu and Dr. Celik (1), which suggested that patients who undergo arthroplasty surgeries should receive regional anesthesia to avoid postoperative cognitive dysfunction (POCD). However, we have some concerns about the strength of their conclusions based on the questionable statistical methods, less details of clinical features, and absent description for the pain of patients and dosage of analgesic drugs in the 2 groups.

First, the study mentioned that there were negative correlations between Mini-Mental State Examination (MMSE) scores measured at postoperative day 7 and the 1-hour intraoperative cortisol measurements. However, the authors combined the data from the general anesthesia and regional anesthesia groups to build the correlativity. For the various anesthesia method, the baseline and variation of cortisol level and MMSE scores are significantly different between the 2 groups (24.24 \pm 5.72 vs. $10.69 \pm 5.18 ; P^{*}=0.044$ ). Thus the anesthesia methods should be considered as confounding factors that would cause the resulting bias.

Second, the study measured cortisol, glucose, insulin, and C-reactive protein levels as the biomarkers to establish the association with POCD. However, glucose metabolism is a complex mechanism, and glucose or insulin levels could be affected by many clinical factors, such as stress of surgery $(2,3)$. Moreover, Sun et al (4) reported that RNA molecular may affect the metabolism of glucose, whereas RNA molecular could also cause POCD $(5,6)$. Additionally, sedative, muscle relaxant, crystalloid solution, and the colloidal solution would be used to maintain the balance and circulation stabilization, which could also cause POCD directly (7). Thus more confounding factors and clinical features should be recorded and considered while analyzing the relationship between the biomarkers and POCD.

Third, with the development of medicine, patients seek more comfort in the treatment process while curing diseases (8). The study did not use any sedation for patients in the regional anesthesia group during the operation. Although all patients received morphine patient-controlled analgesia and additional morphine when necessary, we could not find any details about the pain and the dosage of analgesic drugs in the 2 groups. As is well known that the pain itself and analgesic drugs are critical factors in the development of cognitive dysfunction $(5,9)$. Therefore the postoperative pain and analgesic drugs should be scored and calculated.

Qi Zhao, MD

Department of Anesthesiology and Translational

Neuroscience Center

West China Hospital

Sichuan University

Chengdu, Sichuan, China 610041

E-mail: 544303611@qq.com

Rui Gao, MD

Department of Anesthesiology and Translational Neuroscience Center

West China Hospital

Sichuan University

Chengdu, Sichuan, China 610041

E-mail: sophiegaorui@foxmail.com

Chan Chen, MD, PhD

Department of Anesthesiology and Translational Neuroscience Center

West China Hospital

Sichuan University

Chengdu, Sichuan, China 610041

E-mail: xychenchan@gmail.com

Tao Zhu, MD, PhD

Professor

Department of Anesthesiology and Translational

Neuroscience Center

West China Hospital

Sichuan University

Chengdu, Sichuan, China 610041

E-mail: scuwchtaozhu@foxmail.com 


\section{References}

1. Edipoglu IS, Celik F. The associations between cognitive dysfunction, stress biomarkers, and administered anesthesia type in total knee arthroplasties: Prospective, randomized trial. Pain Physician 2019; 22:495-507.

2. Laaksonen L, Kallioinen M, Langsjo J, et al. Comparative effects of dexmedetomidine, propofol, sevoflurane, and S-ketamine on regional cerebral glucose metabolism in humans: A positron emission tomography study. $\mathrm{Br} J$ Anaesth 2018; 121:281-29o.

3. Mulukutla BC, Yongky A, "Le T", Mashek DG, Hu WS. Regulation of glucose metabolism: A perspective from cell biopro- cessing. Trends Biotechnol 2016; 34:638651.

4. $\quad$ Sun J, Liu Q, Zhao L, et al. Potential reg- 7 . ulation by miRNAs on glucose metabolism in liver of common carp (Cyprinus carpio) at different temperatures. Comp Biochem Physiol Part D Genomics Proteomics 2019; 32:100628.

5. Chen C, Gao R, Li M. Extracellular RNAs$\mathrm{TLR}_{3}$ signaling contributes to cognitive decline in a mouse model of postoperative cognitive dysfunction. Brain Behav Immun 2019; 80:439-451.

6. Li M, Chen C, Zhang W, et al. Identification of the potential key long non-coding RNAs in aged mice with postoper- ative cognitive dysfunction. Front Aging Neurosci 2019; 11:181.

Kletecka J, Holeckova I, Brenkus P, Pouska J, Benes J, Chytra I. Propofol versus sevoflurane anaesthesia: Effect on cognitive decline and event-related potentials. J Clin Monit Comput 2019; 33:665-673.

8. Tawil S, Iskandar K, Salameh P. Pain management in hospitals: Patients' satisfaction and related barriers. Pharm Pract (Granada) 2018; 16:1268.

9. Wang Y, Sands LP, Vaurio L, Mullen EA, Leung JM. The effects of postoperative pain and its management on postoperative cognitive dysfunction. Am J Geriatr Psychiatry 2007; 15:50-59. 